\title{
Attitudes to National Institute for Health and Clinical Excellence guidance on refeeding syndrome
}

\author{
A. N. De Silva ${ }^{1}$, T. R. Smith ${ }^{2}$, M. A. Mullee ${ }^{3}$ and M. A. Stroud ${ }^{1}$ \\ ${ }^{1}$ Institute of Human Nutrition, Southampton University Hospitals, Southampton, UK, ${ }^{2}$ Dept of Gastroenterology, \\ Royal Bournemouth Foundation NHS Trust and ${ }^{3}$ Research \& Development Support Unit, Southampton University Hospitals, \\ Southampton, UK
}

National Institute for Health and Clinical Excellence (NICE) guidelines on nutrition support were issued in $2006^{(1)}$. Those parts relating to refeeding syndrome (RFS) are contentious as they are based on expert opinion rather than clinical evidence. The aim was to characterise UK practice in relation to NICE guidance on RFS.

An electronic questionnaire relating to RFS was sent to a multidisciplinary group of individuals with an interest in nutrition. All members of the following groups dealing with nutritional issues within the UK were included: British Association for Parenteral and Enteral Nutrition Medical (doctors); National Nurses Nutrition Group (nurses); British Pharmaceutical Nutrition Group (pharmacists); Parenteral and Enteral Nutrition Group (dietitians).

In total, 146 individuals (19\%) replied to the 770 questionnaires sent out. Of the responders $72 \%$ felt that NICE guidance had changed their practice, being most notable amongst dietitians (74\%) and least notable amongst doctors (58\%). Only $44 \%$ of doctors as opposed to $70 \%$ of dietitians actually followed NICE guidance. Only $39 \%$ of all responders felt the guidance represented safe practice whilst $36 \%$ felt they were excessively cautious. NICE state that parenteral nutrition (PN) should be started at $50 \%$ of estimated requirements for the first $48 \mathrm{~h}$ feeding. In total $43 \%$ of professionals who believe they follow NICE guidelines start PN at rates $>50 \%$. Perceived incidence of electrolyte abnormalities due to RFS varied markedly, with $4 \%$ feeling they had never seen RFS, whilst $10 \%$ felt they saw RFS in $>20 \%$ of their patients.

NICE guidelines on RFS are contentious to some, partly because they are perceived as adding unnecessary costs to the nutritional care of these patients. They feel that the additional costs of biochemical analyses, close monitoring, electrolyte infusions and slow restoration of nutrients are not justified by any increased risk of harm to patients from RFS.

Others believe that injudicious quick restoration of adequate nutrition leads to RFS or significant hyperglycaemia, which, in addition to potentially harming the patient, may in fact increase hospital costs by prolonging their length of stay as well as incurring added costs relating to the treatment of complications such as electrolyte imbalance, hyperglycaemia or infection.

These data show that practice relating to RFS is split within the UK and highlight the importance of trying to obtain clinical evidence to inform practice when dealing with groups at risk of RFS.

1. National Institute for Health and Clinical Excellence (2006) Nutrition Support in Adults. Clinical Guideline 32. London: NICE. 